\title{
Trajectory of a light ray in Kerr field: A material medium approach
}

\author{
Saswati Roy ${ }^{1,2}$ and A. K. Sen ${ }^{1,3}$
}

\begin{abstract}
The deflection of light ray as it passes around a gravitational mass can be calculated by different methods. Such calculations are generally done by using the null geodesics under both strong field and weak field approximation. However, several authors have studied the gravitational deflection of light ray using material medium approach. For a static, non-rotating spherical mass, one can determine the deflection in Schwarzschild field, by expressing the line element in an isotropic form and calculating the refractive index to determine the trajectory of the light ray. In this paper, we draw our attention to the refractive index of light ray in Kerr field using the material medium approach. The frame dragging effects in Kerr field was considered to calculate the velocity of light ray and finally the refractive index in Kerr field geometry was determined. Hence the deflection of light ray in Kerr field was calculated, assuming far field approximation and compared the results with those calculations done earlier using Null geodesics.
\end{abstract}

Subject headings: Deflection of light ray; Material medium approach; Kerr field geometry; Frame-dragging

\section{Introduction}

Gravitational deflection of light ray is the most important consequence of Einstein's General theory of Relativity. Scientists have worked to find out the light deflection angle due to a gravitating body using different approaches. One of the approaches is the null geodesics, where based on any of the forms of the line element, either by using the perturbation (Misner et al. 1972; Rav d'Inverno 1998) or by integrating the null geodesic equations (Chandrashekhar 1983; Weinberg 1972; Wald 1984), the deflection of light ray is calculated.

\footnotetext{
${ }^{1}$ Physics Department, Assam University, Silchar-788011, India

${ }^{2}$ sr.phy2011@yahoo.com

3asokesen@yahoo.com
} 
In this paper we have considered the material medium approach, to find out the trajectory of the light ray in the gravitational field of a rotating body (also known as Kerr field). In this approach, the effect of the gravitation on the ray of light is estimated by considering the propagation of the electromagnetic waves be reduced to the problem of wave propagation in material medium in flat space time. This concept can be defined as the equivalent material medium approach. This method is attractive because it suggests that the classical optics is as suitable as that of the Riemannian geometry for the study of the electromagnetic phenomena in a gravitational field.

Material medium approach was first used by the author Tamm (1924) and then by Balaz (1958), to calculate the effect of a rotating body on the polarization of light. The same concept had also been utilized by Plebanski (1960) to study the light scattering by gravitational field. Felice (1971) had also discussed the optical phenomena for the deflection of a electromagnetic wave by gravitational field. Mashhoon (1973; 1975) had calculated the deflection and polarization due to the Schwarzschild and Kerr black holes. Later Kopeikin and Mashhoon (2002) also followed the same approach, to correct for the light deflection angle of the Shapiro time delay caused by the rotation of gravitating bodies. Fishbach and Freeman (1980) derived the effective refractive index of the material medium in Schwarzschild field and calculated the second order contribution to the gravitational deflection, where the value of the refractive index of any medium indicates the strength of the gravitational field (Landau \& Lifshitz 1998). In a recent series of articles, Evans and his co-workers (Evans et al. 1986; 1996a; 1996b; Nandi \& Islam 1995) derived and used the effective refractive index to calculate the gravitational time delay and trajectories of light rays in Schwarzschild geometry. The authors also showed that the Opto-mechanical analogy of general relativity reproduces the equation of $\mathbf{G R}$ and matches with the classical equations. P. M. Alsing (1998) has extended the Newtonian formalism of Evans, Nandi and Islam $(1996 b)$ to the case of stationary metrics, typical of rotating space times.

Ishihara, Takahashi and Tmimatsu (1988) studied how the polarization vector of a linearly polarized electromagnetic wave propagates in a curved space-time in the presence of Kerr black hole. They have showed that, in the weak field limit the rotation angle of the plane of polarization is proportional to the line of sight component of the black hole's angular momentum. The above fact is very similar to the Faraday Effect, if the angular momentum is replaced by the magnetic field. This justified that, the rotation of the plane of polarization due to the angular momentum of the Kerr black hole is just the Gravitational Faraday Rotation. Sereno (2003; 2004) has also used the similar idea to derive the time delay function and deflection angle by drawing the trajectory of the light ray by Fermat's principle and also discussed the Gravitational Faraday Rotation in the weak field limit. 
Very recently, Sen (2010) also used the same approach to calculate the light deflection for a static non rotating mass in Schwarzschild geometry, by expressing the line element in an isotropic form to determine the trajectory of the light ray. In this paper we shall follow a similar approach corresponding to Kerr field.

On the other hand, in a more conventional manner, using null geodesic method many authors have worked to find out the deflection angle solutions in the Kerr field. Iyer and Hansen (2009) calculated the deflection angle using the null geodesic in the equatorial plane. They also concluded that the deflection angle due to Kerr field is greater than the Schwarzschild value for pro-grade or direct orbit and smaller for retrograde orbit. But as in the schwarzschild case, when the deflection angle exceeds $2 \pi$, it forms in multiple loops and relativistic images are produced. For the higher rotation case, the effect is much more pronounced.

Bray (1986) presented approximate solutions to the equation of motion for a ray of light in the Kerr field and considered multi-imaging aspect of the gravitational lens effect. Bozza et al.(2005) presented an analytical treatment of gravitational lensing by Kerr black holes, restricting the observers in the equatorial plane. Keeton and Petters $(2005 ; 2006 \mathrm{a} ;$; 2006b) have developed a general formalism for lensing by spherically symmetric lenses and studied the gravitational lensing by compact objects considering the metric in Taylor series expansion in $\frac{G M}{c^{2} r}$. Ye and Lin (2008), discussed the strong similarities between gravitational lensing and optical lensing using the graded refractive index approach.

In the present paper we used the line element under Kerr field in Boyer Lindquist form (1967) to calculate the gravitational bending of a light ray. In Section 2, we have calculated the velocity and hence the refractive index of a light ray in the Kerr field. The value of $\frac{1}{c} \frac{d \phi}{d t}$ involved in the expression of refractive index, due to rotating mass is also evaluated. In Section 3, we have obtained the expression of deflection angle of a light ray in equatorial plane due to Kerr gravitating mass. Section 4 is devoted to make the numerical calculation of the refractive index and deflection angle due to Sun and some other Pulsars. In Section 5, we have concluded our work.

\section{The refractive index of a light ray in Kerr field}

The exact solution of Einstein's Field Equation of General Relativity for a stationary, axially symmetric gravitational field of an uncharged rotating body is given by the Kerr metric. This celebrated solution was first given by R. P. Kerr (Kerr 1963). In the Boyer Lindquist form, in the $(c t, r, \theta, \phi)$ co-ordinate system, the line element of Kerr metric is as 
follows:

$$
\begin{aligned}
d s^{2}=\quad & \left(1-\frac{r_{g} r}{\Sigma^{2}}\right) c^{2} d t^{2}-\frac{\Sigma^{2}}{\Delta} d r^{2}-\Sigma^{2} d \theta^{2}-\left(r^{2}+\alpha^{2}+\frac{r_{g} r \alpha^{2}}{\Sigma^{2}} \sin ^{2} \theta\right) \sin ^{2} \theta d \phi^{2} \\
& +\frac{2 r_{g} r \alpha}{\Sigma^{2}} c \sin ^{2} \theta d \phi d t
\end{aligned}
$$

where

$$
\Sigma^{2}=r^{2}+\alpha^{2} \cos ^{2} \theta
$$

and

$$
\Delta=r^{2}-r_{g} r+\alpha^{2}
$$

The constants $r_{g}$ and $\alpha$ are the Schwarzschild radius and the rotation parameter of the Kerr gravitating body. As usual $r_{g}=\frac{2 G M}{c^{2}}$ and $\alpha$ is defined as $\alpha=\frac{J}{M c}$, where $J$ is the angular momentum of the gravitating body, $\mathrm{M}$ is the total mass of the gravitating body and $c$ is the velocity of light.

If we consider $\alpha \rightarrow 0$ the Kerr line element reduces to Schwarzschild line element, which represents the non-rotating or static gravitating mass. Like the Schwarzschild line element, the Kerr element is also asymptotically flat. The coefficients of line element are independent of $\phi$ showing that it is axially symmetric.

In equatorial plane $\theta=\frac{\pi}{2}$, which implies $\Sigma^{2}=r^{2}$. Under far field approximation we can assume, $\frac{\alpha^{2}}{r^{2}}<<1$. Thus the linearized form of the Kerr metric in terms of spherical polar co-ordinates $r, \theta, \phi$ can be obtained with the first power in $\frac{\alpha}{r}$ as (Islam 2009; Vogt \& Letelier 2005):

$$
\begin{aligned}
d s^{2} & =\left(1-\frac{r_{g}}{r}\right) c^{2} d t^{2}-\frac{1}{\left(1-\frac{r_{g}}{r}\right)} d r^{2}-r^{2} d \theta^{2}-r^{2}\left(1+\frac{\alpha^{2}}{r^{2}}+\frac{r_{g} \alpha^{2}}{r^{3}}\right) d \phi^{2}+\frac{2 r_{g} \alpha}{r} c d \phi d t \\
o r, d s^{2} \cong & {\left[\left(1-\frac{r_{g}}{r}\right)+\frac{2 r_{g} \frac{\alpha}{c}}{r} \frac{d \phi}{d t}\right] c^{2} d t^{2}-\frac{1}{\left(1-\frac{r_{g}}{r}\right)} d r^{2}-r^{2}\left(d \phi^{2}+d \theta^{2}\right) }
\end{aligned}
$$

In most practical purposes the linearized Kerr metric satisfactorily describes the gravitational field around a rotating star or planet. To derive the expression for refractive index, we follow a procedure similar to the one adopted by Sen (2010) for a static field (Schwarzschild geometry). Thus to express the above line element in an isotropic form we introduce a new 
radius co-ordinate $(\rho)$ with the following transformation equation (Landau \& Lifshitz 1998) as

$$
\rho=\frac{1}{2}\left[\left(r-\frac{r_{g}}{2}\right)+r^{1 / 2}\left(r-r_{g}\right)^{1 / 2}\right]
$$

The above equation may be also written as:

$$
r=\rho\left(1+\frac{r_{g}}{4 \rho}\right)^{2}
$$

As was done by Sen (2010) from Eqn. (5) the value of $\frac{d r}{d \rho}$ can be calculated as

$$
\begin{aligned}
\frac{d r}{d \rho} & =\quad\left(1+\frac{r_{g}}{4 \rho}\right)^{2}-\frac{r_{g}}{2 \rho}\left(1+\frac{r_{g}}{4 \rho}\right) \\
& =1-\frac{r_{g}^{2}}{16 \rho^{2}}
\end{aligned}
$$

Substituting the value of $r$ and $d r^{2}$ from Eqn. (55) and (6) in Eqn. (3) (which has far field or slow rotation approximation) we get:

$$
\begin{aligned}
d s^{2} & =\left[1-\frac{r_{g}}{\rho\left(1+\frac{r_{g}}{4 \rho}\right)^{2}}+\frac{2 r_{g} \frac{\alpha}{c}}{\rho\left(1+\frac{r_{g}}{4 \rho}\right)^{2}} \frac{d \phi}{d t}\right] c^{2} d t^{2}-\left[\frac{\left(1-\frac{r_{g}^{2}}{16 \rho^{2}}\right)^{2}}{1-\frac{r_{g}}{\rho\left(1+\frac{r_{g}}{4 \rho}\right)^{2}}}\right] d \rho^{2}-\rho^{2}\left(1+\frac{r_{g}}{4 \rho}\right)^{4}\left(d \phi^{2}+d \theta^{2}\right) \\
& =\left[\frac{\left(1-\frac{r_{g}}{4 \rho}\right)^{2}+2 \frac{r_{g}}{\rho} \frac{\alpha}{c} \frac{d \phi}{d t}}{\left(1+\frac{r_{g}}{4 \rho}\right)^{2}}\right] c^{2} d t^{2}-\left(1+\frac{r_{g}}{4 \rho}\right)^{4}\left[d \rho^{2}+\rho^{2}\left(d \phi^{2}+d \theta^{2}\right)\right]
\end{aligned}
$$

The above result expressed by Eqn.(77) gives the isotropic form of Kerr solution.

Now in spherical co-ordinate system the quantity $\left(d \rho^{2}+\rho^{2}\left(d \phi^{2}+d \theta^{2}\right)\right)$ has the dimension of square of infinitesimal length vector $d \vec{\rho}$.

By setting $d s=0$, the velocity of light $(v(\rho, \theta))$ can be identified from the expression of the form $d s^{2}=f(\rho, \theta) d t^{2}-d \vec{\rho}^{2}$, as $v(\rho, \theta)=\sqrt{f(\rho, \theta)}$. Therefore the velocity of light in the present case (characterized by Schwarzschild radius $r_{g}$ and rotation parameter $\alpha$ ) can be expressed as:

$$
v(\rho, \theta)=\frac{\sqrt{\left(1-\frac{r_{g}}{4 \rho}\right)^{2}+2 \frac{r_{g}}{\rho} \frac{\alpha}{c} \frac{d \phi}{d t}}}{\left(1+\frac{r_{g}}{4 \rho}\right)^{3}} c
$$


But this expression of velocity of light is in the unit of length $\rho$ per unit time. We therefore write

$$
\begin{aligned}
v(r, \theta) & =v(\rho, \theta) \frac{d r}{d \rho} \\
& =v(\rho, \theta)\left[1-\frac{r_{g}^{2}}{16 \rho^{2}}\right] \\
& =c\left(\frac{4 \rho-r_{g}}{4 \rho+r_{g}}\right)^{2} \sqrt{1+8 \frac{\alpha}{c} \frac{d \phi}{d t} \frac{4 \rho r_{g}}{\left(4 \rho-r_{g}\right)^{2}}}
\end{aligned}
$$

Substituting the value of $\rho$ from Eqn. (4) as $4 \rho=2 r-r_{g}+2 \sqrt{r\left(r-r_{g}\right)}$ and then replacing $r / r_{g}$ by $x$, we can write the above expression for velocity of light as :

$$
v^{2}(x, \theta)=\quad c^{2}\left(1-\frac{1}{x}\right)^{2}\left(1+2 \frac{\alpha}{c} \frac{1}{x-1} \frac{d \phi}{d t}\right)
$$

Similarly, the refractive index $n(x, \theta)$ can be expressed by the relation:

$$
n(x, \theta)=\frac{x}{x-1}\left[1+2 \frac{\alpha}{c} \frac{1}{x-1} \frac{d \phi}{d t}\right]^{-\frac{1}{2}}
$$

when $\alpha=0$, we find the central gravitational mass is static and in that case the above expression of refractive index goes over to that for Schwarzschild mass, which is :

$$
n(r)=\frac{x}{x-1}=\frac{r}{r-r_{g}}
$$

This is exactly same as the refractive index calculated by Sen(2010) for a static non rotating mass (Schwarzschild geometry). Thus the second term inside the square bracket of RHS of Eqn. (11) is due to the rotation of the gravitating body. The second term consists of the rotation parameter $\alpha$ and the frame-dragging parameter $\frac{d \phi}{d t}$ due to the rotating body. For a static body, this term just vanishes. However, for a very slow rotating body RHS of Eqn. (11) can be expanded, as an infinite converging series, as the second term is $<<1$. It may be noted here that Sen(2010)'s work is restricted to static geometry. But Eqn. (11) can include, the case of a rotating body (stationary geometry). Therefore, the case discussed by $\operatorname{Sen}(2010)$, comes out as a special case when $\alpha$ is set to be zero. 


\subsection{Calculation of $d \phi / d t$ in the expression of refractive index}

The value of $d \phi / d t$ can be calculated by following a procedure from Landau and Lifshitz

(Landau \& Lifshitz 1998). Below we outline this procedure, which can be used to calculate the expression for $d \phi / d t$.

In the gravitational field of a rotating spherical mass, the relativistic action function $S$ for a particle with the time $t$ and the angle $\phi$ as cyclic variables, can be expressed as:

$$
S=-E_{0} t+L \phi+S_{r}(r)+S_{\theta}(\theta)
$$

where $E_{0}$ is the conserved energy and $L$ denotes the component of the angular momentum along the axis of the symmetry of the field.

The four momentum of the particle is

$$
p^{i}=m c \frac{d x^{i}}{d s}=g^{i k} p_{k}=-g^{i k} \frac{\partial S}{\partial x^{k}}
$$

where $i$ and $k$ have the values $0,1,2,3$ which stand for the coordinates $c t, r, \theta, \phi$ respectively (Landau \& Lifshitz 1998). Now, for the variables $t$ and $\phi$ one can write :

$$
m c \frac{d x^{0}}{d s}=-g^{00} \frac{\partial S}{\partial x^{0}}-g^{01} \frac{\partial S}{\partial x^{1}}-g^{02} \frac{\partial S}{\partial x^{2}}-g^{03} \frac{\partial S}{\partial x^{3}}
$$

and

$$
m c \frac{d x^{3}}{d s}=-g^{30} \frac{\partial S}{\partial x^{0}}-g^{31} \frac{\partial S}{\partial x^{1}}-g^{32} \frac{\partial S}{\partial x^{2}}-g^{33} \frac{\partial S}{\partial x^{3}}
$$

Comparing with the Kerr line element expressed by Eqn. (1) one can write:

$$
\begin{aligned}
& g_{00}=\left(1-\frac{r_{g} r}{\Sigma^{2}}\right), \quad g_{11}=-\frac{\Sigma^{2}}{\Delta}, \quad g_{22}=-\Sigma^{2}, \\
& g_{33}=-\left(r^{2}+\alpha^{2}+\frac{r_{g} r \alpha^{2}}{\Sigma^{2}} \sin ^{2} \theta\right) \sin ^{2} \theta, \\
& g_{03}=g_{30}=\frac{r_{g} r \alpha}{\Sigma^{2}} \sin ^{2} \theta
\end{aligned}
$$

The determinant of the linearized form of the Kerr metric tensor is given by (Landau \& Lifshitz 1998; Wiltshire et al. 2009):

$$
|g|=-\Sigma^{4} \sin ^{2} \theta
$$


Thus using the formula $g^{i j}=\frac{\text { co-factorofg }_{i j}}{|g|}$ the contravariant components are:

$$
\begin{aligned}
& g^{00}=\frac{1}{\Delta}\left(r^{2}+\alpha^{2}+\frac{r_{g} r \alpha^{2}}{\Sigma^{2}} \sin ^{2} \theta\right), \\
& g^{33}=-\frac{\Sigma^{2}-r_{g} r}{\Sigma^{2} \Delta \sin ^{2} \theta} \\
& g^{03}=g^{30}=\frac{r_{g} r \alpha}{\Sigma^{2} \Delta}
\end{aligned}
$$

and other components are zero.

Using the above values of the components of the metric tensors, the Eqn.(15) and (16) become

$$
\begin{gathered}
m c^{2} \frac{d t}{d s}=-\frac{1}{\Delta}\left(r^{2}+\alpha^{2}+\frac{r_{g} r \alpha^{2}}{\Sigma^{2}} \sin ^{2} \theta\right)\left(-\frac{E_{0}}{c}\right)-\frac{r_{g} r \alpha}{\Sigma^{2} \Delta} L \\
m c \frac{d \phi}{d s}=-\frac{r_{g} r \alpha}{\Sigma^{2} \Delta}\left(-\frac{E_{0}}{c}\right)+\frac{\Sigma^{2}-r_{g} r}{\Sigma^{2} \Delta \sin ^{2} \theta} L
\end{gathered}
$$

Therefore, the value of $\frac{d \phi}{d t}$ is

$$
\frac{d \phi}{d t}=\frac{r_{g} r \alpha \sin ^{2} \theta \frac{E_{0}}{c}+\left(\Sigma^{2}-r_{g} r\right) L}{\left[\Sigma^{2}\left(r^{2}+\alpha^{2}+\frac{r_{g} r \alpha^{2}}{\Sigma^{2}} \sin ^{2} \theta\right) \frac{E_{0}}{c}-r_{g} r \alpha L\right] \sin ^{2} \theta} c
$$

In the propagation of a light ray ( or photon like particle), the momentum $(p)$ and the conserved energy $\left(E_{0}\right)$ can be expressed by the relation

$$
E_{0}=p c
$$

In the beginning of our problem, we first considered the general three dimensional form of the Kerr solution. However, to make the problem mathematically simpler to solve, we later defined our geometry contained in the equatorial plane by choosing $\theta=\frac{\pi}{2}$ (in Section 21). By applying such conditions to the original Kerr solution expressed by Eqn.(1), we get a simpler form of the Kerr solution as Eqn.(3). After that we applied the material medium approach to get the value of refractive index as expressed in Eqn.(11). 
But in Section 2.1, to calculate the frame dragging parameter $\frac{d \phi}{c d t}$ of Kerr gravitating body, we have not restricted our work to the equatorial plane in the begining. So we obtained a general expression for frame-dragging as in Eqn.(201).

Later, we calculate the frame-dragging by restricting our problem to the equatorial plane, as it has been already done in Section 2 to make the calculation easy.

Thus, by restricting the light ray in the equatorial plane $\left(\theta=\frac{\pi}{2}\right)$, the angular momentum $L$ can be expressed as

$$
L=p b
$$

where $b=$ impact parameter, which implies

$$
\frac{L c}{E_{0}}=b
$$

So, for equatorial plane, $\Sigma^{2}=r^{2}$ and the Eqn.(20) becomes

$$
\frac{d \phi}{d t}=\frac{r_{g} \alpha+\left(r-r_{g}\right) b}{r^{3}\left(1+\frac{\alpha^{2}}{r^{2}}+\frac{r_{g} r \alpha^{2}}{r^{4}}\right)-r_{g} \alpha b} c
$$

Now under far field approximation (corresponding to the line element as in Eqn. (3)), the above expression becomes

$$
\begin{aligned}
\frac{d \phi}{d t} & =\frac{r_{g} \alpha+\left(r-r_{g}\right) b}{r^{3}-r_{g} \alpha b} c \\
& =\frac{x v+(u-v)}{r_{g}\left(x^{3}-u v\right)} c
\end{aligned}
$$

where we define $\frac{r}{r_{g}}=x$ (as was done earlier), $\frac{\alpha}{r_{g}}=u$ and $\frac{b}{r_{g}}=v$.

Now by substituting the value of $d \phi / d t$ from Eqn.(24) into Eqn. (10), the velocity of propagation of light ray in Kerr geometry can be expressed as :

$$
v^{2}\left(x, \frac{\pi}{2}\right)=\quad c^{2}\left(1-\frac{1}{x}\right)^{2}\left(1+2 \frac{\alpha}{c} \cdot \frac{1}{(x-1)} \cdot \frac{x v+(u-v)}{r_{g}\left(x^{3}-u v\right)} c\right)
$$




$$
\begin{aligned}
& =\quad c^{2}\left(1-\frac{1}{x}\right)^{2}\left(1+2 u \frac{x v+u-v}{(x-1)\left(x^{3}-u v\right)}\right) \\
& =\quad c^{2}\left(1-\frac{1}{x}\right)^{2}+2 u c^{2}\left(1-\frac{1}{x}\right)^{2} \frac{x v+u-v}{(x-1)\left(x^{3}-u v\right)}
\end{aligned}
$$

In the above expression of velocity of light we find, the first term refers to the velocity due to Schwarzschild geometry alone (Sen 2010) and the second term refers to the contribution due to rotation (under Kerr field geometry).

From Eqn.(25), we can write the refractive index $n\left(x, \frac{\pi}{2}\right)$ at an arbitrary point on equatorial plane in the Kerr field as:

$$
\begin{aligned}
n\left(x, \frac{\pi}{2}\right) & =\frac{x}{x-1}\left[1+2 u \frac{x v+u-v}{(x-1)\left(x^{3}-u v\right)}\right]^{-\frac{1}{2}} \\
& =n_{0}(x)\left[1+2 S_{x}\right]^{-\frac{1}{2}} \\
& =n_{0}(x) \cdot \eta_{x}
\end{aligned}
$$

In the above, we introduced the parameters

$$
n_{0}(x)=\frac{x}{x-1}, S_{x}=\frac{u(x v+u-v)}{(x-1)\left(x^{3}-u v\right)} \text { and } \eta_{x}=\left[1+2 S_{x}\right]^{-\frac{1}{2}} .
$$

Here we can also show for all $r>r_{g}$, we get $S_{x}<<1$. This is possible, as when $r>>r_{g}$ and $r>>\alpha$, we must have $x>>1$ and $x>>u$. Also since $\alpha<b$, we must have $u<v$. Now as $x>>1$, we can approximate $(x-1) \sim x$ and then we can finally show that $S_{x}<<1$. Therefore, one can also write the expression of refractive index in terms of the following converging series :

$$
n\left(x, \frac{\pi}{2}\right)=\frac{x}{x-1}\left(1-S_{x}+\frac{3}{2} S_{x}^{2} \ldots\right)
$$

Now at $\alpha=0, S_{x}=0$ so that the refractive index becomes $n\left(x, \frac{\pi}{2}\right)=\frac{x}{x-1}=n_{0}(x)$, which is exactly the same result calculated by Sen(2010). As already discussed in the end of section 2. $n\left(x, \frac{\pi}{2}\right)$ gives the general expression for refractive index in Kerr geometry as a converging infinite series. For a slow rotating body the series will converge much faster. However, for static body the term containing $S_{x}, S_{x}^{2}$ etc. in the RHS of Eqn.(27) will become zero. This will make the refractive index equal to $\frac{x}{x-1}$, which is the expression already obtained by $\operatorname{Sen}(2010)$. 


\section{Calculation of Deflection in Kerr field}

The Eqn. (27) provides the general expression for refractive index on the equatorial plane in Kerr field. Thus, the trajectory of the light ray can be written as (Sen 2010; Born \& Wolf 1959):

$$
\triangle \psi=2 \int_{b}^{\infty} \frac{d r}{r \sqrt{\left(\frac{n(r) . r}{n(b) . b}\right)^{2}-1}}-\pi
$$

As had been already discussed by Sen(2010), here in our present problem the light is approaching from asymptotic infinity $(r=-\infty$ or $x=-\infty)$ towards the rotating gravitational mass, which is placed at the origin and characterized by Schwarzschild radius $r_{g}$ and rotation parameter $\alpha$. Then the ray goes to $r=+\infty$, or $x=+\infty$ after undergoing certain amount of deflection $(\Delta \psi)$. Here, the closest distance of approach, for the approaching ray is $b$. (we note that, in actual case the impact parameter and closet distance of approach are only approximately equal.) When the light ray passes through the closest distance of approach (i.e. $r=b)$, the tangent to the trajectory becomes perpendicular to the vector $\vec{r}($ which is $\vec{b}$ ).

Now we change the variable to $x=\frac{r}{r_{g}}$ so that $d r=r_{g} d x$. The corresponding limit changes from $x=v$ to $x=\infty$, as the limit of $r$ changes from $r=b$ to $r=\infty$.

Accordingly, the value of deflection $(\triangle \psi)$, can be written as :

$$
\begin{aligned}
\triangle \psi & =2 \int_{v}^{\infty} \frac{d x}{x \sqrt{\left(\frac{n(x) \cdot x}{n(v) \cdot v}\right)^{2}-1}}-\pi \\
& =2 I-\pi
\end{aligned}
$$

Using Eqn. (26) and substituting $D_{k}=n(v) \cdot v$, like it was done by Sen $(2010)$, we can re-write the above equation as :

$$
\begin{aligned}
I & =n(v) \cdot v \int_{v}^{\infty} \frac{d x}{x \sqrt{(n(x) \cdot x)^{2}-(n(v) \cdot v)^{2}}} \\
& =D_{k} \int_{v}^{\infty} \frac{d x}{x \sqrt{\left\{n_{0}(x)\left(1+2 S_{x}\right)^{-\frac{1}{2}} \cdot x\right\}^{2}-D_{k}^{2}}} \\
& =D_{k} \int_{v}^{\infty} \frac{d x}{x \sqrt{n_{0}^{2}(x) x^{2}-D_{0}^{2}+n_{0}^{2}(x) x^{2}\left(1+2 S_{x}\right)^{-1}-n_{0}^{2}(x) x^{2}+D_{0}^{2}-D_{k}^{2}}}
\end{aligned}
$$




$$
\begin{aligned}
& =\quad D_{k} \int_{v}^{\infty} \frac{d x}{x \sqrt{n_{0}^{2}(x) x^{2}-D_{0}^{2}}}\left[1+\frac{n_{0}^{2}(x) x^{2}\left\{\left(1+2 S_{x}\right)^{-1}-1\right\}+D_{0}^{2}-D_{k}^{2}}{n_{0}^{2}(x) x^{2}-D_{0}^{2}}\right]^{-\frac{1}{2}} \\
& =\quad D_{k} \int_{v}^{\infty} \frac{d x}{x \sqrt{n_{0}^{2}(x) x^{2}-D_{0}^{2}}}[1+J(x)]^{-\frac{1}{2}}
\end{aligned}
$$

where $D_{0}=n_{0}(v) \cdot v$ (corresponding to schwarzschild deflection). And we have also denoted

$$
J(x)=\frac{n_{0}^{2}(x) x^{2}\left\{\left(1+2 S_{x}\right)^{-1}-1\right\}+D_{0}^{2}-D_{k}^{2}}{n_{0}^{2}(x) x^{2}-D_{0}^{2}}
$$

At this stage we can show that $J(x)<<1$. As $J(x)$ is discontinuous at $x= \pm v$, we can remove its discontinuity and evaluate its values by applying L'Hospital's rule, which are as follows :

$J(x=+v)=\frac{u\{2 u-v(v-2)(v-1)\}}{(v-2)\left\{2 u^{2}+(v-1)\left(v^{3}+u v\right)\right\}}-\frac{u v(v-1)\left(4 v^{3}-3 v^{2}+u v\right)\{u+(v-1) v\}}{(v-2)\left\{2 u^{2}+(v-1)\left(v^{3}+u v\right)\right\}^{2}}$

and

$J(x=-v)=-\frac{u\{2 u+v(v+2)(v+1)\}}{(v+2)\left\{2 u^{2}+(v+1)\left(v^{3}+u v\right)\right\}}-\frac{u v(v+1)\left(4 v^{3}+3 v^{2}+u v\right)\{u+(v+1) v\}}{(v+2)\left\{2 u^{2}+(v+1)\left(v^{3}+u v\right)\right\}^{2}}$

Further, by differentiating $J(x)$ and subsequently applying L'Hospital's rule once again, it can be shown that $J(x)$ has maxima at $x=+v$ and $x=-v$ and all other values of $J(x)$ within the domain $x=+\infty$ to $x=-\infty$ are less than this maxima. These maxima values are tabulated in Table 1. We may note that, these values are much smaller than 1 and actually become still smaller compared to 1 , when either $x>>v$ or the field is weaker or both. So we may safely assume that, $J(x)<<1$ for all $x$, for all practical purposes. In Table 1 , we find for Sun the difference in $J(x)$ values for $x=+v$ and $x=-v$, occurs only at 16th place after the decimal. For other objects we report $J(x)$ values up to 5 th place after the decimal.

Therefore, from Eqns.(29) and (30) one can write:

$$
\begin{array}{ccc}
\triangle \psi & =2 D_{k} \int_{v}^{\infty} \frac{d x}{x \sqrt{n_{0}^{2}(x) x^{2}-D_{0}^{2}}}\left[1-\frac{1}{2} J(x)+\frac{3}{8} J^{2}(x)-\frac{5}{16} J^{3}(x)+\frac{35}{128} J^{4}(x)-\frac{63}{254} J^{5}(x)+\ldots \ldots . .\right]-\pi \\
& = & 2\left[I_{0}+I_{1}+I_{2}+I_{3}+\ldots \ldots \ldots \ldots . .\right]-\pi
\end{array}
$$


where, we have introduced additional notations:

$$
\begin{gathered}
I_{0}=D_{k} \int_{v}^{\infty} \frac{d x}{x \sqrt{n_{0}^{2}(x) x^{2}-D_{0}^{2}}} \\
I_{1}=D_{k} \int_{v}^{\infty} \frac{d x}{x \sqrt{n_{0}^{2}(x) x^{2}-D_{0}^{2}}}(-1 / 2 J(x)) \\
I_{2}=D_{k} \int_{v}^{\infty} \frac{d x}{x \sqrt{n_{0}^{2}(x) x^{2}-D_{0}^{2}}}\left(3 / 8 J^{2}(x)\right)
\end{gathered}
$$

and so on.

Again we follow a procedure same as what was followed by $\operatorname{Sen}(2010)$ to evaluate a similar integral.

Thus

$$
\begin{aligned}
I_{0} & =D_{k} \int_{v}^{\infty} \frac{d x}{x \sqrt{\left(\frac{x}{x-1}\right)^{2} x^{2}-D_{0}^{2}}} \\
& =D_{k} \int_{v}^{\infty} \frac{(x-1) d x}{x^{3} \sqrt{1-\frac{D_{0}^{2}(x-1)^{2}}{x^{4}}}} \\
& =D_{k} \int_{v}^{\infty} \frac{(x-2) d x}{x^{3} \sqrt{1-\frac{D_{0}^{2}(x-1)^{2}}{x^{4}}}}+D_{k} \int_{v}^{\infty} \frac{d x}{x^{3} \sqrt{1-\frac{D_{0}^{2}(x-1)^{2}}{x^{4}}}} \\
& =I_{01}+I_{02}(\text { say })
\end{aligned}
$$

The first part in above (viz $I_{01}$ ) contains an integral, which has been already evaluated earlier by Sen (2010). Now we introduce a new variable $y=D_{0} x^{-2}(x-1)$, so that $d y=$ $-D_{0} x^{-3}(x-2) d x$. Therefore, the limits of integration change as $y=0$ and

$$
\begin{aligned}
y & =\quad D_{0} \cdot v^{-2}(v-1) \\
& =\quad n_{0}(v) \cdot v \cdot v^{-2}(v-1) \\
& =\frac{v}{v-1} \cdot v \cdot v^{-2}(v-1) \\
& =1
\end{aligned}
$$


Table 1: Values of $S_{x}$ and $J(x)$ of different Gravitating Objects for $x=-v$ and $x=+v$

\begin{tabular}{|c|c|c|c|c|}
\hline Name of Objects & $S_{x}(x=-v)$ & $S_{x}(x=+v)$ & $J(x=-v)$ & $J(x=+v)$ \\
\hline SUN & $0.10241529958672695 \mathrm{e}-10$ & $0.10241529958672697 \mathrm{e}-10$ & $0.307243 \mathrm{e}-10$ & $0.307248 \mathrm{e}-10$ \\
\hline $\begin{array}{l}\text { PSR B 1919+21 } \\
\text { (Hewish et el. } 1968 \text { ) }\end{array}$ & 0.02720094 & 0.02749950 & 0.05714 & 0.12267 \\
\hline $\begin{array}{l}\text { PSR J 1748-2446 ad } \\
\text { (Hessel et al. 2006) }\end{array}$ & 0.02503882 & 0.02528190 & 0.05342 & 0.11053 \\
\hline $\begin{array}{l}\text { PSR B 1937+21 } \\
\text { (Ashworthet al. } 1983 \text { ) }\end{array}$ & 0.02234780 & 0.02254167 & 0.04809 & 0.09890 \\
\hline $\begin{array}{l}\text { PSR J 1909-3744 } \\
\text { (Jacoby et al. } 2003 \text { ) }\end{array}$ & 0.01235494 & 0.01239606 & 0.02940 & 0.04735 \\
\hline PSR 1855+09 & 0.00630369 & 0.00631039 & 0.01633 & 0.02181 \\
\hline $\begin{array}{l}\text { PSRJ 0737-3039 A } \\
\text { (Lvne et al. 2004) }\end{array}$ & 0.00146678 & 0.00146691 & 0.00417 & 0.00461 \\
\hline PSR 0531+21 & 0.00100644 & 0.00100648 & 0.00289 & 0.00313 \\
\hline $\begin{array}{l}\text { PSR B 1534+12 } \\
\text { (Stairs et al. 1998) }\end{array}$ & 0.00087751 & 0.00087755 & 0.00252 & 0.00273 \\
\hline
\end{tabular}


Thus,

$$
\begin{aligned}
I_{01} & =D_{k} \int_{v}^{\infty} \frac{(x-2) d x}{x^{3} \sqrt{1-\frac{D_{0}^{2}(x-1)^{2}}{x^{4}}}} \\
& =\frac{D_{k}}{D_{0}} \int_{0}^{1} \frac{d y}{\sqrt{1-y^{2}}} \\
& =\frac{D_{k}}{D_{0}} \cdot \frac{\pi}{2}
\end{aligned}
$$

In the expression for $I_{02}$, if we change the variable as $z=\frac{1}{x}$, then the limits of this integration changes from $z=\frac{1}{v}=a($ say $)$ to $z=0$. A a result, we get a form of integral as obtained by Sen in his Eqn.(17) (2010). We can therefore, write:

$$
\begin{aligned}
I_{02} & =D_{k} \int_{v}^{\infty} \frac{d x}{x^{3} \sqrt{1-\frac{D_{0}^{2}(x-1)^{2}}{x^{4}}}} \\
& =D_{k} \int_{v}^{\infty} \frac{d x}{\sqrt{x^{6}-D_{0}^{2} x^{2}(x-1)^{2}}} \\
& =D_{k} \int_{0}^{a} \frac{z d z}{\sqrt{1-D_{0}^{2} z^{2}(1-z)^{2}}}
\end{aligned}
$$

This can be evaluated in terms of Elliptical function as expressed by Eqn.(18) of Sen(2010). And finally for a given value of $a$, its numerical value can be obtained.

Now to evaluate the value of $J(x)$ we evaluate the value of $\left(\left(1+2 S_{x}\right)^{-1}-1\right)$ first as follows:

$$
\begin{aligned}
\left(1+2 S_{x}\right)^{-1}-1 & =\frac{1}{1+2 S_{x}}-1 \\
& =-\frac{\frac{2 u(x v+u-v)}{(x-1)\left(x^{3}-u v\right)}}{1+\frac{2 u(x v+u-v)}{(x-1)\left(x^{3}-u v\right)}} \\
& =-\frac{2 u(x v+u-v)}{(x-1)\left(x^{3}+u v\right)+2 u^{2}}
\end{aligned}
$$

Now in Eqn. (31) above, we substitute the value of $\left(\left(1+2 S_{x}\right)^{-1}-1\right)$ (from Eqn. (37)) and $n_{0}(x)=x /(x-1)$. As a result, we can write the following expressions for $J(x)$ : 


$$
\begin{aligned}
J(x) & =\frac{n_{0}^{2}(x) x^{2}\left\{\left(1+2 S_{x}\right)^{-1}-1\right\}+D_{0}^{2}-D_{k}^{2}}{n_{0}^{2}(x) x^{2}-D_{0}^{2}} \\
& =\frac{n_{0}^{2}(x) x^{2}\left\{\left(1+2 S_{x}\right)^{-1}-1\right\}+D_{0}^{2}-D_{0}^{2}\left(1+2 S_{v}\right)^{-1}}{n_{0}^{2}(x) x^{2}-D_{0}^{2}} \\
& =\frac{\frac{x^{4}}{(x-1)^{2}}\left\{-\frac{2 u(x v+u-v)}{(x-1)\left(x^{3}+u v\right)+2 u^{2}}\right\}+D_{0}^{2}\left\{\frac{2 u\left(v^{2}+u-v\right)}{(v-1)\left(v^{3}+u v\right)+2 u^{2}}\right\}}{\frac{x^{4}}{(x-1)^{2}}-D_{0}^{2}} \\
& =\frac{-x^{4}\left\{\frac{2 u(x v+u-v)}{(x-1)\left(x^{3}+u v\right)+2 u^{2}}\right\}+D_{0}^{2}(x-1)^{2}\left\{\frac{2 u\left(v^{2}+u-v\right)}{(v-1)\left(v^{3}+u v\right)+2 u^{2}}\right\}}{x^{4}-D_{0}^{2}(x-1)^{2}}
\end{aligned}
$$

Further, substituting the value of $n_{0}(x)=\frac{x}{x-1}$ and $J(x)$ from Eqn. (38), the integral $I_{1}$ becomes

$$
\begin{aligned}
I_{1} & =\quad-\frac{1}{2} D_{k} \int_{v}^{\infty} \frac{J(x)}{x \sqrt{n_{0}^{2} x^{2}-D_{0}^{2}}} d x \\
& =\quad-\frac{1}{2} D_{k} \int_{v}^{\infty} \frac{1}{x \sqrt{\frac{x^{4}}{(x-1)^{2}}-D_{0}^{2}}}\left[\frac{-x^{4}\left\{\frac{2 u(x v+u-v)}{(x-1)\left(x^{3}+u v\right)+2 u^{2}}\right\}+D_{0}^{2}(x-1)^{2}\left\{\frac{2 u\left(v^{2}+u-v\right)}{x^{4}-D_{0}^{2}(x-1)^{2}}\right\}}{\left.x^{3}+u v\right)+2 u^{2}}\right] d x \\
& =-\frac{1}{2} D_{k} \int_{v}^{\infty} \frac{(x-1)}{\sqrt{x^{6}-D_{0}^{2} x^{2}(x-1)^{2}}}\left[\frac{-x^{4}\left\{\frac{2 u(x v+u-v)}{(x-1)\left(x^{3}+u v\right)+2 u^{2}}\right\}+D_{0}^{2}(x-1)^{2}\left\{\frac{2 u\left(v^{2}+u-v\right)}{(v-1)\left(v^{3}+u v\right)+2 u^{2}}\right\}}{x^{4}-D_{0}^{2}(x-1)^{2}}\right] d x
\end{aligned}
$$

Again applying the change of variable as $z=\frac{1}{x}$ (like it was done for $I_{02}$ ) we may write the integral $I_{1}$ as

$$
I_{1}=-\frac{1}{2} D_{k} \int_{0}^{a} \frac{(1-z)}{\sqrt{1-D_{0}^{2} z^{2}(1-z)^{2}}}\left[\frac{-\left\{\frac{2 u z^{3}(v+(u-v) z)}{(1-z)\left(1+u v z^{3}\right)+2 u^{2} z^{4}}\right\}+D_{0}^{2} z^{2}(1-z)^{2}\left\{\frac{2 u\left(v^{2}+u-v\right)}{(v-1)\left(v^{3}+u v\right)+2 u^{2}}\right\}}{1-D_{0}^{2} z^{2}(1-z)^{2}}\right] d z
$$

Similarly, $I_{2}, I_{3}$ etc. can be written as

$$
I_{2}=\frac{3}{8} D_{k} \int_{0}^{a} \frac{(1-z)}{\sqrt{1-D_{0}^{2} z^{2}(1-z)^{2}}}\left[\frac{-\left\{\frac{2 u z^{3}(v+(u-v) z)}{(1-z)\left(1+u v z^{3}\right)+2 u^{2} z^{4}}\right\}+D_{0}^{2} z^{2}(1-z)^{2}\left\{\frac{2 u\left(v^{2}+u-v\right)}{(v-1)\left(v^{3}+u v\right)+2 u^{2}}\right\}}{1-D_{0}^{2} z^{2}(1-z)^{2}}\right]^{2} d z
$$




$$
\begin{aligned}
& I_{3}=\quad-\frac{5}{16} D_{k} \int_{0}^{a} \frac{(1-z)}{\sqrt{1-D_{0}^{2} z^{2}(1-z)^{2}}}\left[\frac{-\left\{\frac{2 u z^{3}(v+(u-v) z)}{(1-z)\left(1+u v z^{3}\right)+2 u^{2} z^{4}}\right\}+D_{0}^{2} z^{2}(1-z)^{2}\left\{\frac{2 u\left(v^{2}+u-v\right)}{\left.(v-1) v^{3}+u v\right)+2 u^{2}}\right\}}{1-D_{0}^{2} z^{2}(1-z)^{2}}\right]^{3} d z \\
& I_{4}=\quad \frac{35}{64} D_{k} \int_{0}^{a} \frac{(1-z)}{\sqrt{1-D_{0}^{2} z^{2}(1-z)^{2}}}\left[\frac{-\left\{\frac{2 u z^{3}(v+(u-v) z)}{(1-z)\left(1+u v z^{3}\right)+2 u^{2} z^{4}}\right\}+D_{0}^{2} z^{2}(1-z)^{2}\left\{\frac{2 u\left(v^{2}+u-v\right)}{(v-1)\left(v^{3}+u v\right)+2 u^{2}}\right\}}{1-D_{0}^{2} z^{2}(1-z)^{2}}\right]^{4} d z \\
& I_{5}=\quad-\frac{63}{256} D_{k} \int_{0}^{a} \frac{(1-z)}{\sqrt{1-D_{0}^{2} z^{2}(1-z)^{2}}}\left[\frac{-\left\{\frac{2 u z^{3}(v+(u-v) z)}{(1-z)\left(1+u v z^{3}\right)+2 u^{2} z^{4}}\right\}+D_{0}^{2} z^{2}(1-z)^{2}\left\{\frac{2 u\left(v^{2}+u-v\right)}{(v-1)\left(v^{3}+u v\right)+2 u^{2}}\right.}{1-D_{0}^{2} z^{2}(1-z)^{2}}\right]^{5} d z
\end{aligned}
$$

Thus form Eqn. (32), the expression for deflection of light ray in Kerr geometry can be expressed as:

$$
\begin{aligned}
& \triangle \psi=2\left[\frac{D_{k}}{D_{0}} \frac{\pi}{2}+D_{k}\left\{\int_{0}^{a} \frac{z d z}{\sqrt{1-D_{0}^{2} z^{2}(1-z)^{2}}}\right.\right. \\
& -\frac{1}{2} \int_{0}^{a} \frac{(1-z)}{\sqrt{1-D_{0}^{2} z^{2}(1-z)^{2}}}\left(\frac{-\left\{\frac{2 u z^{3}(v+(u-v) z)}{(1-z)\left(1+u v z^{3}\right)+2 u^{2} z^{4}}\right\}+D_{0}^{2} z^{2}(1-z)^{2}\left\{\frac{2 u\left(v^{2}+u-v\right)}{(v-1)\left(v^{3}+u v\right)+2 u^{2}}\right\}}{1-D_{0}^{2} z^{2}(1-z)^{2}}\right) d z \\
& +\frac{3}{8} \int_{0}^{a} \frac{(1-z)}{\sqrt{1-D_{0}^{2} z^{2}(1-z)^{2}}}\left(\frac{-\left\{\frac{2 u z^{3}(v+(u-v) z)}{(1-z)\left(1+u v z^{3}\right)+2 u^{2} z^{4}}\right\}+D_{0}^{2} z^{2}(1-z)^{2}\left\{\frac{2 u\left(v^{2}+u-v\right)}{(v-1)\left(v^{3}+u v\right)+2 u^{2}}\right\}}{1-D_{0}^{2} z^{2}(1-z)^{2}}\right)^{2} d z \\
& -\frac{5}{16} \int_{0}^{a} \frac{(1-z)}{\sqrt{1-D_{0}^{2} z^{2}(1-z)^{2}}}\left(\frac{-\left\{\frac{2 u z^{3}(v+(u-v) z)}{(1-z)\left(1+u v z^{3}\right)+2 u^{2} z^{4}}\right\}+D_{0}^{2} z^{2}(1-z)^{2}\left\{\frac{2 u\left(v^{2}+u-v\right)}{(v-1)\left(v^{3}+u v\right)+2 u^{2}}\right\}}{1-D_{0}^{2} z^{2}(1-z)^{2}}\right)^{3} d z \\
& +\frac{35}{64} \int_{0}^{a} \frac{(1-z)}{\sqrt{1-D_{0}^{2} z^{2}(1-z)^{2}}}\left[\frac{-\left\{\frac{2 u z^{3}(v+(u-v) z)}{(1-z)\left(1+u v z^{3}\right)+2 u^{2} z^{4}}\right\}+D_{0}^{2} z^{2}(1-z)^{2}\left\{\frac{2 u\left(v^{2}+u-v\right)}{(v-1)\left(v^{3}+u v\right)+2 u^{2}}\right\}}{1-D_{0}^{2} z^{2}(1-z)^{2}}\right]^{4} d z \\
& -\frac{63}{256} \int_{0}^{a} \frac{(1-z)}{\sqrt{1-D_{0}^{2} z^{2}(1-z)^{2}}}\left[\frac{-\left\{\frac{2 u z^{3}(v+(u-v) z)}{(1-z)\left(1+u v z^{3}\right)+2 u^{2} z^{4}}\right\}+D_{0}^{2} z^{2}(1-z)^{2}\left\{\frac{2 u\left(v^{2}+u-v\right)}{(v-1)\left(v^{3}+u v\right)+2 u^{2}}\right\}}{1-D_{0}^{2} z^{2}(1-z)^{2}}\right]^{5} d z \\
& +\ldots \ldots . . .\}]-\pi
\end{aligned}
$$




$$
\begin{aligned}
o r, \triangle \psi= & \frac{D_{k}}{D_{0}} \pi+2 D_{k}\left[\int_{0}^{a} \frac{z d z}{\sqrt{1-D_{0}^{2} z^{2}(1-z)^{2}}}\right. \\
& -\frac{1}{2} \int_{0}^{a} \frac{(1-z)}{\sqrt{1-D_{0}^{2} z^{2}(1-z)^{2}}}\left(\frac{-\left\{\frac{2 u z^{3}(v+(u-v) z)}{(1-z)\left(1+u v z^{3}\right)+2 u^{2} z^{4}}\right\}+D_{0}^{2} z^{2}(1-z)^{2}\left\{\frac{2 u\left(v^{2}+u-v\right)}{(v-1)\left(v^{3}+u v\right)+2 u^{2}}\right\}}{1-D_{0}^{2} z^{2}(1-z)^{2}}\right) d z \\
& +\frac{3}{8} \int_{0}^{a} \frac{(1-z)}{\sqrt{1-D_{0}^{2} z^{2}(1-z)^{2}}}\left(\frac{-\left\{\frac{2 u z^{3}(v+(u-v) z)}{(1-z)\left(1+u v z^{3}\right)+2 u^{2} z^{4}}\right\}+D_{0}^{2} z^{2}(1-z)^{2}\left\{\frac{2 u\left(v^{2}+u-v\right)}{(v-1)\left(v^{3}+u v\right)+2 u^{2}}\right\}}{1-D_{0}^{2} z^{2}(1-z)^{2}}\right)^{2} d z \\
& -\frac{5}{16} \int_{0}^{a} \frac{(1-z)}{\sqrt{1-D_{0}^{2} z^{2}(1-z)^{2}}}\left(\frac{-\left\{\frac{2 u z^{3}(v+(u-v) z)}{(1-z)\left(1+u v z^{3}\right)+2 u^{2} z^{4}}\right\}+D_{0}^{2} z^{2}(1-z)^{2}\left\{\frac{2 u\left(v^{2}+u-v\right)}{(v-1)\left(v^{3}+u v\right)+2 u^{2}}\right\}}{1-D_{0}^{2} z^{2}(1-z)^{2}}\right) \\
& +\frac{35}{64} \int_{0}^{a} \frac{(1-z)}{\sqrt{1-D_{0}^{2} z^{2}(1-z)^{2}}}\left[\frac{-\left\{\frac{2 u z^{3}(v+(u-v) z)}{(1-z)\left(1+u v z^{3}\right)+2 u^{2} z^{4}}\right\}+D_{0}^{2} z^{2}(1-z)^{2}\left\{\frac{2 u\left(v^{2}+u-v\right)}{(v-1)\left(v^{3}+u v\right)+2 u^{2}}\right\}}{1-D_{0}^{2} z^{2}(1-z)^{2}}\right]^{4} d z \\
& -\frac{63}{256} \int_{0}^{a} \frac{(1-z)}{\sqrt{1-D_{0}^{2} z^{2}(1-z)^{2}}}\left[\frac{-\left\{\frac{2 u z^{3}(v+(u-v) z)}{(1-z)\left(1+u v z^{3}\right)+2 u^{2} z^{4}}\right\}+D_{0}^{2} z^{2}(1-z)^{2}\left\{\frac{2 u\left(v^{2}+u-v\right)}{(v-1)\left(v^{3}+u v\right)+2 u^{2}}\right\}}{1-D_{0}^{2} z^{2}(1-z)^{2}}\right]^{5} d z \\
& +\ldots \ldots . .]-\pi
\end{aligned}
$$

The above expression has been obtained for gravitational deflection in the equatorial plane of a rotating body considering the Kerr line element.

\section{Calculation of numerical values for deflection for some Gravitational objects}

Considering Sun as a rotating body, we can calculate the deflection angle for pro-grade $(b=-v e)$ and retro-grade $(b=+v e)$ direction of the light ray with respect to the gravitating body. From Eqn.(45) after actual numerical calculations, it was found that for pro-grade direction, the deflection will be greater and for retro-grade direction, the deflection will be smaller than that of the Schwarzschild geometry. This has been also confirmed by Iyer et al. in their previous work (2009) and also by Alsing (1998) in a similar work. A more recent work by Werner (2012), also confirms this phenomena where the author has considered terms only up to first order in $\alpha$ ( rotation parameter).

For a Sun grazing ray, we can calculate the deflection of a light ray using the Eqn.(45) obtained in this present work. Here, we may consider the closest distance of approach is equal to the solar radius as $r_{\odot}=6.955 \times 10^{5} \mathrm{~km}$, solar mass as $M_{\odot}=1.99 \times 10^{30} \mathrm{~kg}$ and solar time period as $T=28$ days. 
In addition to Sun, we shall also consider some millisecond pulsars, to show the effect of rotation on light deflections, as we know the pulsars are fast rotating objects. Nunez et al. (2010) have calculated the red-shift and preferred radius for some fast millisecond pulsars. We derive some of the input parameters ( like $b, \alpha, r_{g}$ etc.) from their published work and perform some sample calculations. We note that, the calculations performed here on Sun and these pulsars are for demonstrative purpose only. The aim is to show that, like the Null Geodesic method, the Material Medium approach can predict equally well the gravitational deflection of the light ray in Kerr field.

In table 2, we have shown the refractive index along with other parameters for different gravitating body, due to pro-grade and retro-grade orbits of the light ray. The refractive index values have been expressed with 11 places after the decimal. We can note for Sun the refractive index value changes at 11th place after the decimal as we change from pro-grade to retro-grade orbit of light ray. However, for fast rotating pulsars these refractive index values vary at the second place after the decimal between pro-grade and retrograde orbits ( cf. Table 2). From this table it is totally clear that, the refractive index is greater for pro-grade direction and smaller for retro-grade direction as compared to Schwarzschild one. Thus as compared to the Schwarzschild case, the deflection angle for light ray should be also greater for pro-grade and smaller for retro-grade orbits of light ray.

In table 3, we have calculated the deflection of a light ray (in arc-sec) due to Sun and other pulsars considering the impact parameter as the physical radius of the gravitating body and considering up to fifth order term i.e. $I_{5}$ in the Eqns. (32) and (45). We further, note that Sun is a slow rotating object and pulsars are fast rotating. To calculate the deflection angle of these rotating gravitational objects, we used numerical integration by Simpson's one third rule. Here it is also shown that, the value of deflection angle continuously decreases as we calculate higher order terms. In this table the deflection angle values due to the gravitational body are calculated considering no rotation $(\alpha=0)$ of the body, pro-grade $(b=-v e)$ and retro-grade $(b=+v e)$ orbits of light ray. In case of no rotation $(\alpha=0)$, the first column ( representing $\left(2 I_{0}-\pi\right)$ ) will be zero as there is no difference between $D_{k}$ and $D_{0}$. Also the values of $I_{1}, I_{2}, I_{3}$ etc. will be zero, when there is no rotation (i.e. $\alpha=0$ ) as can be seen in Table 3 and equally confirmed through Eqn (45). The light deflection values obtained for Sun, by material medium method here for pro-grade and retro-grade orbits are in good agreement with those other similar calculations done using null geodesic method [Iyer \& Hansen(2009)] . 
Table 2. Refractive index due to different Gravitational Objects.

\begin{tabular}{|c|c|c|c|c|c|c|c|c|}
\hline $\begin{array}{l}\text { Name of the } \\
\text { Gravitational } \\
\text { Objects }\end{array}$ & $\begin{array}{l}\text { Schwarzs } \\
\text { child } \\
\text { Radius } \\
r_{g}(K m)\end{array}$ & $\begin{array}{l}\text { Rotation } \\
\text { Parameter } \\
\alpha(K m)\end{array}$ & $\begin{array}{l}\text { Physical } \\
\text { Radius } R \\
\text { or } \\
b(K m)\end{array}$ & $\begin{array}{l}u \\
\left(\alpha / r_{g}\right)\end{array}$ & $\begin{array}{l}v \\
\left(b / r_{g}\right)\end{array}$ & $\begin{array}{l}\text { Refractive } \\
\text { index } \mathrm{n} \\
(\alpha=0)\end{array}$ & $\begin{array}{l}\text { Refractive } \\
\text { index n } \\
\text { (Pro-grade) } \\
(b=-v e)\end{array}$ & $\begin{array}{l}\text { Refractive } \\
\text { index n } \\
\text { (Retro-grade) } \\
(b=+v e)\end{array}$ \\
\hline$S U N$ & 2.9554 & 1.6762 & $6.955 \mathrm{e}+05$ & 0.5671 & 235331.2911 & 1.00000424934 & 1.00000424935 & 1.00000424933 \\
\hline $\begin{array}{l}P S R B 1919+21 \\
\text { (Hewish et el. 1968) }\end{array}$ & 4.1375 & 2.5075 & $2.000 \mathrm{e}+01$ & 0.6060 & 4.8337 & 1.26084097201 & 1.29285456990 & 1.22753613865 \\
\hline $\begin{array}{l}\text { PSRJ } 1748-2446 \\
\text { (Hessel et al. 2006) }\end{array}$ & 3.9898 & 2.4261 & $2.010 \mathrm{e}+01$ & 0.6080 & 5.0378 & 1.24765683468 & 1.27698068171 & 1.21726163358 \\
\hline $\begin{array}{l}P S R B 1937+21 \\
\text { (Ashworthet al. 1983) }\end{array}$ & 3.9898 & 2.1970 & $2.020 \mathrm{e}+01$ & 0.5506 & 5.0629 & 1.24612905064 & 1.27244504736 & 1.21895467231 \\
\hline $\begin{array}{l}\text { PSRJ } 1909-3744 \\
\text { (Jacoby et al. } 2003 \text { ) }\end{array}$ & 4.2498 & 2.7486 & $3.110 \mathrm{e}+01$ & 0.6467 & 7.3178 & 1.15828146469 & 1.17215555862 & 1.14418491095 \\
\hline$P S R 1855+09$ & 3.9898 & 3.4339 & $4.690 \mathrm{e}+01$ & 0.8606 & 11.7549 & 1.09298024705 & 1.09976248166 & 1.08614772138 \\
\hline $\begin{array}{l}P S R J 0737-3039 \\
\text { (Lyne et al. } 2004)\end{array}$ & 3.9602 & 6.5918 & $1.336 \mathrm{e}+02$ & 1.6645 & 33.7352 & 1.03054808987 & 1.03205416015 & 1.02903968046 \\
\hline$P S R 0531+21$ & 3.9898 & 6.8042 & $1.644 \mathrm{e}+02$ & 1.7054 & 41.2050 & 1.02487248899 & 1.02590137268 & 1.02384251987 \\
\hline $\begin{array}{l}\text { PSRB } 1534+12 \\
\text { (Stairs et al. } 1998 \text { ) }\end{array}$ & 3.9602 & 6.0879 & $1.659 \mathrm{e}+02$ & 1.5372 & 41.8913 & 1.02445506278 & 1.02535206812 & 1.02355723286 \\
\hline
\end{tabular}


Table 3. Deflection of a light ray due to Sun and some Pulsars

\begin{tabular}{|c|c|c|c|c|c|c|c|c|}
\hline $\begin{array}{l}\text { Name } \\
\text { of } \\
\text { Objects }\end{array}$ & $\begin{array}{c}2 I_{01}-\pi \\
(\mathrm{a}) \\
(\text { arc-sec })\end{array}$ & $\begin{array}{c}2 I_{02} \\
(\mathrm{~b}) \\
(\operatorname{arc}-\mathrm{sec})\end{array}$ & $\begin{array}{c}2 I_{1} \\
(\mathrm{c}) \\
(\operatorname{arc}-\mathrm{sec})\end{array}$ & $\begin{array}{c}2 I_{2} \\
(\mathrm{~d}) \\
(\operatorname{arc}-\mathrm{sec})\end{array}$ & $\begin{array}{c}2 I_{3} \\
(\mathrm{e}) \\
(\operatorname{arc}-\mathrm{sec})\end{array}$ & $\begin{array}{c}2 I_{4} \\
(\mathrm{f}) \\
(\operatorname{arc}-\mathrm{sec})\end{array}$ & $\begin{array}{c}2 I_{5} \\
(\mathrm{~g}) \\
(\operatorname{arc}-\mathrm{sec})\end{array}$ & $\begin{array}{c}\text { Total Deflection } \\
\begin{array}{c}(\mathrm{a}+\mathrm{b}+\ldots+\mathrm{f}+\mathrm{g}) \\
\triangle \phi(\operatorname{arc}-\mathrm{sec})\end{array}\end{array}$ \\
\hline $\begin{array}{l}\text { Sun } \\
\alpha=0\end{array}$ & 0.00000 & 1.752008089976 & & & & & & 1.752008089976 \\
\hline $\begin{array}{l}\text { Pro } \\
(b=-\mathrm{ve})\end{array}$ & $0.66364 \mathrm{e}-05$ & 1.752008089994 & $-0.18122 \mathrm{e}-05$ & $0.10665 \mathrm{e}-16$ & $-0.40393 \mathrm{e}-28$ & $0.12069 \mathrm{e}-38$ & $-0.50226 \mathrm{e}-50$ & 1.752012914194 \\
\hline $\begin{array}{l}\text { Retro } \\
(b=+\mathrm{ve})\end{array}$ & $-0.66365 \mathrm{e}-05$ & 1.752008089959 & $-0.18122 \mathrm{e}-05$ & $0.10665 \mathrm{e}-16$ & $-0.76563 \mathrm{e}-28$ & $0.12069 \mathrm{e}-38$ & $-0.50226 \mathrm{e}-50$ & 1.751999641259 \\
\hline $\begin{array}{l}\mathbf{B 1 9 1 9} \\
\alpha=0\end{array}$ & 0.00000 & 1119735.4072 & & & & & & 119735.4072 \\
\hline $\begin{array}{l}\text { Pro } \\
\text { (b=-ve) }\end{array}$ & 16453.1545 & 122775.5695 & -10484.7664 & 307.7444 & -5.3394 & 0.8887 & $-0.1876 \mathrm{e}-01$ & 129047.2325 \\
\hline $\begin{array}{l}\text { Retro } \\
(b=+\mathrm{ve})\end{array}$ & -17116.7756 & 116572.6231 & -9955.0483 & 292.1964 & -10.5747 & 0.8438 & $-0.1781 \mathrm{e}-01$ & 89783.2468 \\
\hline $\begin{array}{l}\mathbf{J 1 7 4 8} \\
\alpha=0\end{array}$ & 0.00000 & $\overline{c 112974.7176}$ & & & & & & 1112974.7176 \\
\hline $\begin{array}{l}\text { Pro } \\
(b=-\mathrm{ve})\end{array}$ & 15230.0314 & 115629.9776 & -9290.5744 & 243.6810 & -3.7975 & 0.5612 & $-0.1057 \mathrm{e}-01$ & 121809.8687 \\
\hline $\begin{array}{l}\text { Retro } \\
(b=+\mathrm{ve})\end{array}$ & -15786.4644 & 110222.4469 & -8856.0931 & 232.2850 & $\begin{array}{l}-7.5082 \\
\end{array}$ & 0.5350 & $-0.1008 \mathrm{e}-01$ & 85805.1911 \\
\hline $\begin{array}{l}\mathbf{B 1 9 3 7} \\
\alpha=0\end{array}$ & 0.00000 & 112197.2094 & & & & & & 1112197.2094 \\
\hline $\begin{array}{l}\text { Pro } \\
\text { (b=-ve) }\end{array}$ & 13684.5905 & 114566.6120 & -8286.1164 & 194.5455 & -2.7143 & 0.3591 & $-0.6060 \mathrm{e}-02$ & 120157.2703 \\
\hline $\begin{array}{l}\text { Retro } \\
(b=+\mathrm{ve})\end{array}$ & -14130.9579 & 109750.5211 & -8751.2927 & 226.4006 & -7.2176 & 0.5072 & $\begin{array}{c}-0.9428 \mathrm{e}-02 \\
\end{array}$ & 87087.9512 \\
\hline $\begin{array}{l}\mathbf{J 1 9 0 9} \\
\alpha=0\end{array}$ & 0.00000 & 69386.1605 & & & & & & "69386.1605 \\
\hline $\begin{array}{l}\text { Pro } \\
\text { (b=-ve) }\end{array}$ & 7761.8550 & 70217.2798 & -3538.9772 & 37.3630 & -0.2419 & $0.1382 \mathrm{e}-01$ & $\begin{array}{l}-0.1043 \mathrm{e}-03 \\
\end{array}$ & 74477.2924 \\
\hline $\begin{array}{l}\text { Retro } \\
(b=+\mathrm{ve})\end{array}$ & $\begin{array}{l}-7886.3101 \\
\end{array}$ & 68541.7148 & -3454.5281 & 36.4714 & -0.4728 & $0.1349 \mathrm{e}-01$ & $-0.1008 \mathrm{e}-03$ & 57236.8885 \\
\hline $\begin{array}{l}\mathbf{1 8 5 5} \\
\alpha=0\end{array}$ & 0.00000 & 39691.1980 & & & & & & 396691.1980 \\
\hline Pro & 4021.0132 & 39937.4925 & -1486.8124 & 6.8396 & $-0.1971 \mathrm{e}-01$ & $0.4775 \mathrm{e}-03$ & $-0.1563 \mathrm{e}-05$ & 42478.5136 \\
\hline
\end{tabular}




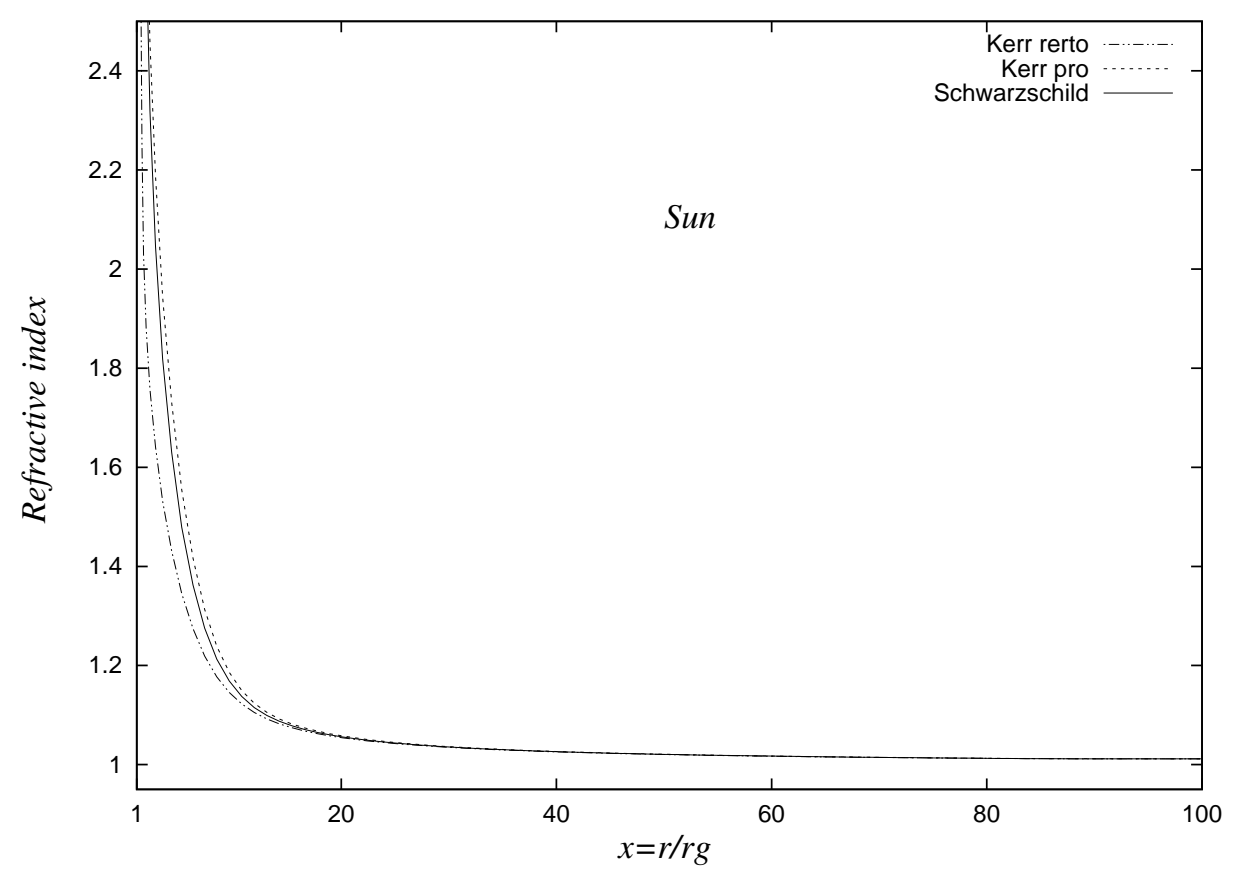

Fig. 1.- Refractive index $\left(n\left(x, \frac{\pi}{2}\right)\right)$ as a function of $x\left(=\frac{r}{r_{g}}\right)$ for Sun.

In Fig 1 we have plotted the refractive index as a function of $x$ considering the gravitational body to be Sun. If we make this plot for other pulsars, we get the similar trend in the curves. As the value of $x$ i.e. $\frac{r}{r_{g}}$ increases, (i.e. as we move towards asymptotically flat space), these curves merge into each other. At $x=1$ i.e. $r=r_{g}$, the value of refractive index is infinite for all the gravitational bodies and this is physically expected.

In Fig,2, we have plotted the deflection angle as a function of $u\left(=\frac{\alpha}{r_{g}}\right)$ for Sun. Thus, Fig:2 illustrates the dependence of deflection angle on the rotation parameter. Here the solid line parallel to the $u$-axis, indicates the Schwarzschild geometry.

The pulsars are highly compact objects, with their physical radii being very close to the corresponding Schwarzschild radii in most of the cases. Thus it is necessary to consider the light ray to be passing at various other close distances from the pulsars. In order to understand the dependence of deflection on the impact parameter, we selected one of the pulsars from our list viz. PSRB 1919+21. In Fig 3 we have plotted the amount of deflection as a function of $v=b / r_{g}$, under three cases no-rotation, pro-grade orbit and retro-grade orbit. From Fig, 3, it is also clear that the deflection angle is greater for pro-grade and smaller for retro-grade orbit as compared to the Schwarzschild one. This phenomena has been also observed by Iyer et al. (2009) as discussed earlier.

From Fig,2 and 3, and also from Table 3, it is clear that the deviations of light orbit 
Table 3-Continued

\begin{tabular}{|c|c|c|c|c|c|c|c|c|}
\hline $\begin{array}{l}\text { Name } \\
\text { of } \\
\text { Objects }\end{array}$ & $\begin{array}{c}2 I_{01}-\pi \\
(\mathrm{a}) \\
(\operatorname{arc}-\mathrm{sec})\end{array}$ & $\begin{array}{c}2 I_{02} \\
(\mathrm{~b}) \\
(\operatorname{arc}-\mathrm{sec})\end{array}$ & $\begin{array}{c}2 I_{1} \\
(\mathrm{c}) \\
(\operatorname{arc}-\mathrm{sec})\end{array}$ & $\begin{array}{c}2 I_{2} \\
(\mathrm{~d}) \\
(\operatorname{arc}-\mathrm{sec})\end{array}$ & $\begin{array}{c}2 I_{3} \\
(\mathrm{e}) \\
(\operatorname{arc}-\mathrm{sec})\end{array}$ & $\begin{array}{c}2 I_{4} \\
(\mathrm{f}) \\
(\operatorname{arc}-\mathrm{sec})\end{array}$ & $\begin{array}{c}2 I_{5} \\
(\mathrm{~g}) \\
(\operatorname{arc}-\mathrm{sec})\end{array}$ & $\begin{array}{c}\text { Total Deflection } \\
(\mathrm{a}+\mathrm{b}+\ldots+\mathrm{f}+\mathrm{g}) \\
\triangle \phi(\text { arc-sec })\end{array}$ \\
\hline \multicolumn{9}{|l|}{$(b=-\mathrm{ve})$} \\
\hline $\begin{array}{l}\text { Retro } \\
(b=+\mathrm{ve})\end{array}$ & -4050.8295 & 39443.0772 & -1468.4061 & 6.7549 & $-0.3805 e-01$ & $0.4715 \mathrm{e}-03$ & $-0.1543 e-05$ & 33930.5589 \\
\hline $\begin{array}{l}\mathbf{J 0 7 3 7} \\
\alpha=0\end{array}$ & 0.00000 & 12734.8996 & & & & & & 12734.8996 \\
\hline $\begin{array}{l}\text { Pro } \\
(b=-\mathrm{ve})\end{array}$ & 947.0043 & 12753.5108 & -285.9891 & 0.2616 & $-0.1527 \mathrm{e}-03$ & $0.7180 \mathrm{e}-06$ & $-0.4655 \mathrm{e}-09$ & 13414.7874 \\
\hline $\begin{array}{l}\text { Retro } \\
(b=+\mathrm{ve})\end{array}$ & -948.4751 & 12716.2596 & -285.1537 & 0.2608 & $-0.2915 \mathrm{e}-03$ & $0.7159 \mathrm{e}-06$ & $-0.4642 \mathrm{e}-09$ & 11482.8913 \\
\hline $\begin{array}{l}\mathbf{0 5 3 1} \\
\alpha=0\end{array}$ & 0.00000 & 10347.3213 & & & & & & 10347.3213 \\
\hline $\begin{array}{l}\text { Pro } \\
(b=-\mathrm{ve})\end{array}$ & 650.5361 & 10357.7091 & -192.8309 & 0.1193 & $-0.4718 \mathrm{e}-04$ & $0.1496 \mathrm{e}-06$ & $-0.6561 \mathrm{e}-010$ & 10815.5335 \\
\hline $\begin{array}{l}\text { Retro } \\
(b=+\mathrm{ve})\end{array}$ & -651.2224 & 10336.9225 & -192.4439 & 0.1190 & $-0.8998 \mathrm{e}-04$ & $0.1493 \mathrm{e}-06$ & $-0.6548 \mathrm{e}-010$ & 9493.3751 \\
\hline $\begin{array}{l}\mathbf{1 5 3 4} \\
\alpha=0 \\
\end{array}$ & 0.00000 & 10172.1154 & & & & & & 10172.1154 \\
\hline $\begin{array}{l}\text { Pro } \\
(b=-\mathrm{ve})\end{array}$ & 567.3840 & 10181.0220 & -167.9483 & $0.9054 \mathrm{e}-01$ & $-0.3119 \mathrm{e}-04$ & $0.8622 \mathrm{e}-07$ & $-0.3293 \mathrm{e}-010$ & 10580.5482 \\
\hline $\begin{array}{l}\text { Retro } \\
(b=+\mathrm{ve})\end{array}$ & -567.9056 & 10163.2006 & -167.6543 & $0.9038 \mathrm{e}-01$ & $-0.5950 \mathrm{e}-04$ & $0.8607 \mathrm{e}-07$ & $-0.3287 \mathrm{e}-010$ & 9427.7310 \\
\hline
\end{tabular}




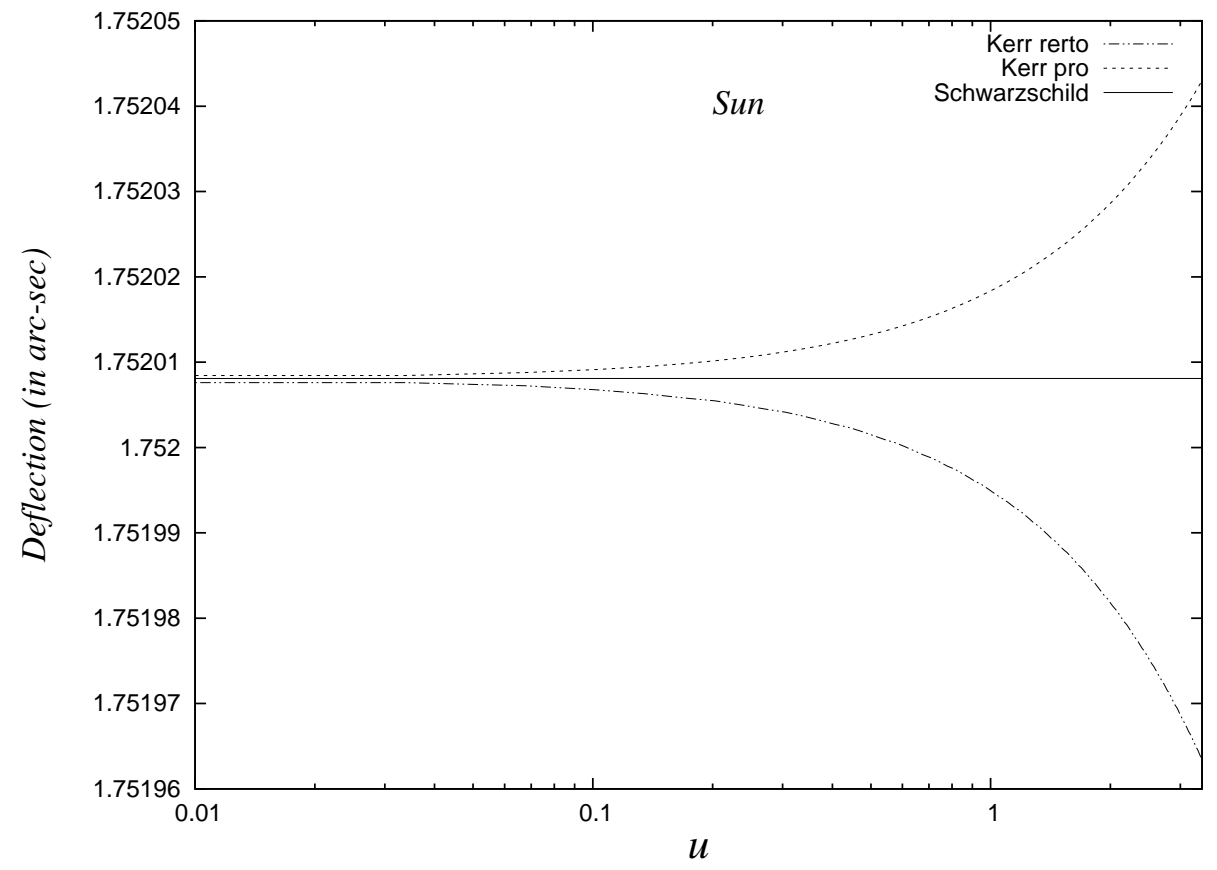

Fig. 2.- Deflection $(\triangle \psi)$ as a function of $u\left(=\frac{\alpha}{r_{g}}\right)$ for Sun.

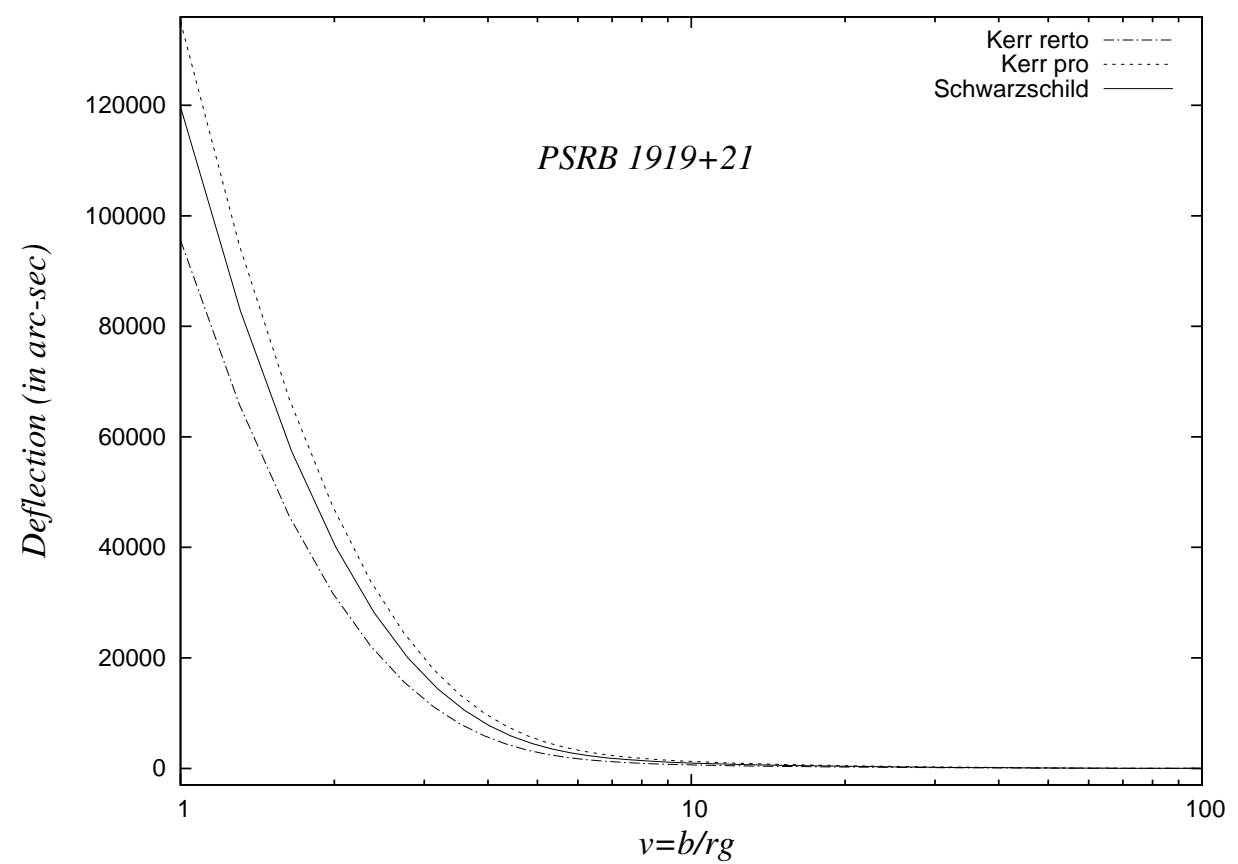

Fig. 3.- Deflection $(\triangle \psi)$ as a function of $v\left(=\frac{b}{r_{g}}\right)$ for pulsar $P S R B 1919+21$. 
from the Schwarzschild case for pro-grade and retro-grade orbit are not symmetric. Such asymmetries of deflection values for pro-grade and retro-grade orbits have been also reported by previous authors [Iyer(2009)].

\section{Conclusions}

We have presented here in detail the calculations for light deflection angle on the equatorial plane of a rotating objects (viz Kerr field), by following material medium approach. The following have been observed:

(i) The material medium approach gives same values of deflection as compared to that obtained by other most conventional method of Null geodesic. This has been verified by taking Sun as a test case. Further, the cases of some millisecond pulsars were considered to understand the effect of rotation more objectively on the deflection angle.

(ii) For pro-grade orbit of the deflection angle is greatest and for retro-grade orbit, the deflection angle is smallest. The one corresponding to no-rotation ( Schwarzschild case) lies in between.

(iii) The deviations of pro-grade and retro-grade orbits from the Schwarzschild deflection angle are not symmetric. The deviation is slightly higher for retro-garde orbit.

\section{Acknowledgments}

We are thankful to anonymous reviewer of this paper for his/her useful comments.

\section{REFERENCES}

Alsing, P. M.: Am. J. Phys. 66, 779 (1998)

Ashworth, M., Lyne, A. G., Smith, F.G.: Nature 301, 313 (1983)

Balazs, N. L.: Phys. Rev. 110, 236 (1958)

Born M., Wolf, E.: Principles of Optics (7th Edition, Cambridge University Press, Cambridge,1999), p121 (1959)

Boyer, R. H., Lindquist, R. W.: J. Math. Phys. 8, 265 (1967) 
Bozza, V., Luca, F. de, Scarpetta, G., Sereno, M.: Phys. Rev. D 72, 083003 (2005)

Bray, I.: Phys. Rev. D 34, 367 (1986)

Chandrasekhar, S.: The Mathematical Theory of Black Holes, Oxford University Press, New York (1983)

Evans, J., Rosenquist, M.: Am. J. Phys. 54, 876 (1986)

Evans, J., Nandi, K. K., Islam, A.: Am. J. Phys. 64, 1404 (1996a)

Evans, J., Nandi, K. K., Islam, A.: Gen. Relativ. Gravit. 28, 413 (1996b)

Felice, F. de: Gen. Relativ. Gravit. 2, 347 (1971)

Fischbach, E., Freeman, B. S.: Phys. Rev. D 22, 2950 (1980)

Hessels, J. W. T., Ransom, S. M., Stairs, I. H., Freire, P. C. C., Kaspi, V. M., Camilo, F.: Science, 311, 1901 (2006)

Hewish, A., Bell, S. J., Pilkington, J. D. H., Scott, P. F., Collins, R. A.: Nature, 217, 709 (1968)

Ishihara, H., Takahashi, M., Tomimatsu, A.: Phys. Rev. D 38, 472 (1988)

Islam, J. N.: Rotating Fields in General Relativity, Cambridge University Press, New York (2009)

Iyer, S. V., Hansen, E. C.: Phys. Rev. D 80, 124023 (2009)

Jacoby, B. A., Bailes, M., Kerkwijk, M. H. van, Ord, S., Hotan, A., Kulkarni, S. R., Anderson, S. B.: The Astrophysical J. 599, L99 (2003)

Keeton, C. R., Petters, A.O.: Phys. Rev. D 72, 104006 (2005)

Keeton, C. R., Petters, A.O.: Phys. Rev. D 73, 044024 (2006a)

Keeton, C. R., Petters, A.O.: Phys. Rev. D 73, 104032 (2006b)

Kerr, R. P.: Phys. Rev. Letters, 11, 237 (1963)

Kopeikin, S., Mashhoon, B.: Phys. Rev. D 65, 064025 (2002)

Landau, J. L. D., Lifshitz, E. M.: The Classical Theory of Fields, volume 2, (1st edition Pergamon Press, 1951) 4th English edition Butterworth-Heinemann (1998) p17,22,28,246,324 
Lyne, A. G., Burgay, M., Kramer, M.: Science, 303, 1153 (2004)

Mashhoon, B.: Phys. Rev. D 7, 2807 (1973)

Mashhoon, B.: Phys. Rev. D 11, 2679 (1975)

Misner, C. W., Thorne, K. S., Wheeler, J. A.: Gravitation, W. H. Freeman and Company, New York (1971)

Nandi, K. K., Islam, A.: Am. J. Phys. 63, 251 (1995)

Nunez, P. D., Nowakowski, M.: J. Astrophys. Astr. 31, 105 (2010)

Plebanski, J.: Phys. Rev. 118, 1396 (1960)

Ray, d'Inverno: Introducing Einstein's Relativity, Oxford University Press, New York(Reprint) (1998)

Sen, A. K.: Astrophysics 53, 560 (2010)

Sereno, M.: Phys. Rev. D 67, 064007 (2003)

Sereno, M.: Phys. Rev. D 69, 087501 (2004)

Stairs, I. H., Arzoumanian, Z., Camilo, F., Lyne, A. G., Nice, D. J., Taylor, J. H., Thorsett, S. E., Wolszczan, A.: The Astrophysical J. 505, 352 (1998)

Tamm, J. E.: J. Russ. Phys.-Chem. Soc. 56, 284 (1924)

Vogt, D., Letelier, P. S.: Mon. Not. R. Astron. Soc. 363, 268 (2005)

Wald, R. M.: General Relativity, University of Chicago Press, Chicago and London (1984)

Weinberg, S.: Gravitation and Cosmology, John Wiley \& Sons Inc., New York (1972)

Werner, M. C.: Gen. Relativ. Gravit., 44, 3047 (2012)

Wiltshire, D. L., Visser, M., Scott, S. M.: The Kerr Spacetime, Cambridge University Press, New York, p16 (2009)

Ye, X. H., Lin, Q.: J. Mod. Opt. 55, 1119 (2008) 